OPEN ACCESS

Edited by:

Pietro Daniele Spanu, Imperial College London, UK

Reviewed by:

Raffaella Balestrini, Consiglio Nazionale delle Ricerche,

Marc Tad Nishimura

Colorado State University, Fort Collins, USA

${ }^{*}$ Correspondence: Jun Guo

guojunwgq@nwsuaf.edu.cn;

Zhensheng Kang

kangzs@nwsuaf.edu.cn

tThese authors have contributed equally to this work.

Specialty section: This article was submitted to Plant Biotic Interactions, a section of the journal Frontiers in Plant Science

Received: 18 March 2016 Accepted: 02 June 2016

Published: 21 June 2016

Citation:

Liu P, Myo T, Ma W, Lan D, Qi T, Guo J, Song P, Guo J and Kang Z (2016) TaTypA, a Ribosome-Binding GTPase Protein, Positively Regulates Wheat Resistance to the Stripe Rust

Fungus. Front. Plant Sci. 7:873. doi: 10.3389/fp/s.2016.00873

\section{TaTypA, a Ribosome-Binding GTPase Protein, Positively Regulates Wheat Resistance to the Stripe Rust Fungus}

\author{
Peng Liut, Thwin Myo+, Wei Ma, Dingyun Lan, Tuo Qi, Jia Guo, Ping Song, Jun Guo *and \\ Zhensheng Kang *
}

State Key Laboratory of Crop Stress Biology for Arid Areas, College of Plant Protection, Northwest A\&F University, Yangling, China

Tyrosine phosphorylation protein A (TypA/BipA) belongs to the ribosome-binding GTPase superfamily. In many bacterial species, TypA acts as a global stress and virulence regulator and also mediates resistance to the antimicrobial peptide bactericidal permeability-increasing protein. However, the function of TypA in plants under biotic stresses is not known. In this study, we isolated and functionally characterized a stressresponsive TypA gene (TaTypA) from wheat, with three copies located on chromosomes 6A, 6B, and 6D, respectively. Transient expression assays indicated chloroplast localization of TaTypA. The transcript levels of TaTypA were up-regulated in response to treatment with methyl viologen, which induces reactive oxygen species (ROS) in chloroplasts through photoreaction, cold stress, and infection by an avirulent strain of the stripe rust pathogen. Knock down of the expression of TaTypA through virusinduced gene silencing decreased the resistance of wheat to stripe rust accompanied by weakened ROS accumulation and hypersensitive response, an increase in TaCAT and TaSOD expression, and an increase in pathogen hyphal growth and branching. Our findings suggest that TaTypA contributes to resistance in an ROS-dependent manner.

Keywords: TypA, Puccinia striiformis f. sp. tritici, ROS, virus-induced gene silencing, Triticum aestivum, tyrosine phosphorylation

\section{INTRODUCTION}

During the growth of plants that are subjected to different environmental stresses, including drought, salinity, chilling, and metal toxicity as well as pathogen attack. In these biotic and abiotic stresses, reactive oxygen species (ROS) are generated in plants due to a disruption of cellular homeostasis (Sharma and Dubey, 2005; Srivastava and Dubey, 2011). ROS are comprised of free radicals, such as superoxide anion $\left(\mathrm{O}_{2}{ }^{-}\right)$and hydroxyl radical $(\cdot \mathrm{OH})$, as well as nonradical molecules, like hydrogen peroxide $\left(\mathrm{H}_{2} \mathrm{O}_{2}\right)$ and singlet oxygen $\left({ }^{1} \mathrm{O}_{2}\right.$; Sharma and Dubey, 2005). Diverse mechanisms have been shown to be involved in pathogen-induced ROS production, including peroxidases and different oxidases (oxalate oxidase, amine oxidase, and NADPH oxidase; Pugin et al., 1997; Wojtaszek, 1997; Rea et al., 1998). The increased production of ROS during biotic and abiotic stresses can pose a threat to plant cells by causing oxidation of proteins, peroxidation of lipids, damage to nucleic acids, inhibition of enzymes, activation of the programmed cell death (PCD) pathway, and ultimately lead to death of the cells. ROS act as more likely cofactors in redox reactions taking part in various roles in plant defenses (Torres, 2010). For example, ROS have been characterized as primary signaling molecules modulating multiple physiological processes during 
plant growth and development (De Tullio, 2010). Intriguingly, evolutionary considerations based on the NADPH gene family imply that mechanisms detoxifying ROS were acquired before the plants used ROS as signaling molecules (Mittler et al., 2011). The reasons that make ROS important signaling regulators are: (i) rapid control over the production and scavenging of ROS in individual cells, allowing a dynamic control of ROS levels; (ii) accumulation of ROS in different subcellular organelles, resulting in an efficient intracellular control; (iii) rapid propagation of ROS-induced signaling from the origin of the stimuli to nearby cells; (iv) interaction and modification of a variety of targets by ROS (Mittler et al., 2011). Remarkably, plants exploit this versatility of ROS when responding to the environment and during biotic interactions (Scheler et al., 2013).

Tyrosine phosphorylation protein A (TypA/BipA) is a member of the ribosome-binding GTPase superfamily. GTPases are widely distributed molecular switches found across all bacterial species (Robinson, 2008) and are involved in the regulation of multiple cellular processes, including protein translocation, translation, tRNA modification, ribosome biogenesis and assembly, cell polarity, cell division and diverse signaling events (Verstraeten et al., 2011). Translational GTPases (trGTPases) are involved in GTPase activity that is induced by the large ribosomal subunit of bacterial species (Ramakrishnan, 2002; Nilsson and Nissen, 2005; Margus et al., 2007). A few members of trGTPases family, such as EF-G, EF-Tu, IF2 and RF3, bind to an overlapping site on the ribosome (Sergiev et al., 2005). TypA/BipA was firstly identified in Salmonella typhimurium as a protein induced by the antimicrobial peptide bactericidal permeability-increasing protein (BPI; Qi et al., 1995). Previous studies suggested that TypA has an effect on expression of the global regulator Fis by destabilizing unusually strong interactions between the $5^{\prime}$-untranslated region of fis mRNA and the ribosome. TypA binding to ribosomes at a site in accordance with that of EF-G indicated that GTPase activity of TypA is sensitive to high GDP:GTP ratios (Owens et al., 2004). Several studies showed that TypA is involved in regulating diverse virulence-related mechanisms in Escherichia coli, including flagella-mediated cell motility and cytoskeletal rearrangements in host epithelial cells (Farris et al., 1998), capsule synthesis and expression of genes from different pathogenicity islands (Grant et al., 2003). TypA is necessary for survival of Sinorhizobium meliloti under some stress conditions, such as low $\mathrm{pH}$, low temperature, and treatment with sodium dodecyl sulfate (SDS; Kiss et al., 2004) and is required for growth of E. coli K12 at low temperatures (Pfennig and Flower, 2001). TypA are widely distributed in plants and are present in green algae, mosses, liverworts, gymnosperms, and angiosperms (Barak and Trebitsh, 2007). Recently, Atkinson (2015) identified a previously unreported TypA subfamily (mTypA) which are mitochondrially targeted according to transit peptide predictions in some Archaeplastida, Amoebozoa, and fungi (Atkinson, 2015). TypA genes also have roles in pollen tube growth in Arabidopsis (Lalanne et al., 2004) and in development of male reproductive organs in cucumber (Barak and Trebitsh, 2007).
SsTypA was proposed as a new member of the ROS-scavenging system in the chloroplast of Suaeda salsa when subjected to environmental stress (Wang et al., 2008). However, little is known about the functions of TypA gene in plant defense against pathogens.

Wheat stripe rust, caused by Puccinia striiformis f. sp. tritici (Pst), is a destructive disease of wheat (Triticum aestivum) worldwide (Hau and de Vallavieille-Pope, 2006). The fungus is a strict biotroph and is dependent on living host cells for growth and survival. This life style has hindered study of the molecular mechanisms involved in the wheat-Pst interaction. In a previous study, to isolate defense-related genes against the fungus, we constructed an incompatible suppression subtractive hybridization ( $\mathrm{SSH}$ ) cDNA library of wheat leaves infected by Pst and identified a cDNA fragment exhibiting high homology to the rice TypA protein (Yu et al., 2010). In the present work, we identified a wheat TypA homolog, designated TaTypA. The transcript profile of TaТурA was analyzed in wheat seedlings inoculated with Pst and in plants subjected to environmental stimuli, and the subcellular localization of TaTypA was determined. Furthermore, knock down of TaTypA in wheat was performed to analyze whether and how TaTypA participates in resistance to stripe rust. Our results demonstrated that ТаТурA performs a positive function in resistance by regulating ROS-induced signaling.

\section{MATERIALS AND METHODS}

\section{Plant Materials, Fungal Pathogens, and Treatments}

Wheat cv. Suwon 11 (Su11) and isolates of Pst pathotypes CYR23 (avirulent) and CYR31 (virulent) were used in the study. Su11, carrying the $\mathrm{YrSu}$ resistance gene, expresses a typical hypersensitive response (HR) to CYR23, but is susceptible to CYR31 (Cao et al., 2002). Wheat seedlings were grown, inoculated and maintained following procedures and conditions described previously (Kang et al., 2002). For RNA isolation wheat leaves were collected at $0,12,24,36,48,72,96$, and 120 h postinoculation (hpi) with CRY23 or CYR31. The time points were selected based on a microscopic study of the interactions between Su11 with CYR23 and CYR31 (Kang et al., 2002; Wang et al., 2007). A methyl viologen (MV) concentration gradient assay was conducted to induce oxidative stress, whereby 10-days-old wheat seedlings were sprayed with $0.1,1$, or $5 \mathrm{mM} \mathrm{MV}$. Samples were collected at $0,4,6,8,12$, and $24 \mathrm{~h}$ hpt. Control wheat seedlings were treated with sterile distilled water in both treatments. Tendays-old wheat seedlings were placed in a $4^{\circ} \mathrm{C}$ chamber for low-temperature treatment. Roots of 10-days-old wheat seedlings were soaked in 20\% PEG 6000 for the drought stress treatment, or in $200 \mathrm{mM} \mathrm{NaCl}$ to induce salt stress. The first leaves of the treated and control plants treated with sterile distilled water were collected at $0,6,12$, and $24 \mathrm{hpt}$. Three biological replicates were included in each assay. Tissues harvested from all treatments were immediately frozen in liquid nitrogen and stored at $-80^{\circ} \mathrm{C}$ until the extraction of total RNA. 


\section{Identification and Sequence Analysis of the TaTypA Gene}

A 433-bp unisequence (GenBank accession number EV253998) exhibiting high homology to a putative TypA Oryza sativa GTP-binding protein was obtained from the Pst-induced SSH cDNA library (Yu et al., 2010) and used as a query sequence to screen EST databases of wheat constructed in our laboratory (Ma et al., 2009). Homologous wheat EST clones were retrieved and assembled, and according to the assembled sequence, we designed the TaTypA-F and TaTypA-R primers (Supplementary Table S1) to amplify TaTypA. The amplified product was cloned into the pGEM-T Easy Vector (Promega, Madison, WI, USA) for sequencing. This cDNA product was aligned with the wheat cv. Chinese Spring (CS) genome using data of the International Wheat Genome Sequencing Consortium ${ }^{1}$. The predicted chromosomal location and related sequences were also obtained from this website. The cDNA sequences were analyzed with ORF Finder ${ }^{2}$ and the BLAST ${ }^{3}$ programs. The amino acid sequence was analyzed with $\mathrm{Pfam}^{4}$, InterProScan ${ }^{5}$ and PROSITE Scan ${ }^{6}$ to identify conserved domains. Multiple sequence alignment was performed with ClustalW2.0 (Chenna et al., 2003) and DNAMAN7.0 software (Lynnon Biosoft, USA), and a phylogenetic tree of alignment of amino acid sequences was generated with Mega 5.0 software (Tamura et al., 2011).

\section{RNA Extraction, cDNA Synthesis, and qRT-PCR}

Total RNA was extracted with the Trizol ${ }^{\mathrm{TM}}$ Reagent (Invitrogen, Carlsbad, CA, USA) following the manufacturer's instructions. Three $\mu \mathrm{g}$ of RNA were subjected to first strand cDNA synthesis with the Promega RT-PCR system (Promega, Madison, WI, USA) and Oligo (dT) 18 primer. Relative quantification of TaTypA expression was performed with a SYBR Green qRT-PCR mixture on an ABI prism 7500 sequence detection system (Applied Biosystems, USA). Specific primers (Supplementary Table S1) were designed and qRT-PCR was conducted according to previously described procedures (Wang et al., 2009). The wheat

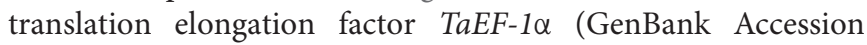
number Q03033) was used as an internal reference for normalization. Dissociation curves were produced for each reaction to ensure specific amplification. Threshold $\mathrm{Ct}$ values were generated from the ABI PRISM 7500 Software Tool (Applied Biosystems) to quantify relative gene expression. Transcript levels of TaTypA were calculated by the comparative $2^{-\Delta \Delta C T}$ method (Livak and Schmittgen, 2001). Transcript abundance was assessed from three independent biological replicates.

\footnotetext{
${ }^{1}$ http://wheat-urgi.versailles.inra.fr/Seq-Repository/BLAST

${ }^{2}$ http://www.ncbi.nlm.nih.gov/gorf/gorf.html

${ }^{3}$ http://www.ncbi.nlm.nih.gov/blast/

${ }^{4}$ http://pfam.xfam.org/

${ }^{5}$ http://www.ebi.ac.uk/interpro/search/sequence-search

${ }^{6}$ http://prosite.expasy.org/scanprosite/
}

\section{Subcellular Location of TaTypA-GFP Fusion Protein and Western Blotting}

Protoplasts for subcellular localization of TaTypA protein were isolated from mesophyll tissue of 1-week-old wheat seedlings as previously described (Li et al., 2015). A recombinant plasmid, pCaMV35S::TaTypA::GFP, was constructed by cloning fulllength cDNA and of TaTypA into a pCaMV35S::GFP vector by PCR using primers TaTyPA-163-F and TaTyPA-163-R (Supplementary Table S1). For analysis of the chloroplast transit peptide in TaTypA, the partial coding sequence (positions 1-240, $240 \mathrm{nt}$ ) at N-terminal was fused into pCaMV35S::GFP vector by PCR using primers TaTyPA-163-F and TaTyPA-163- $\mathrm{R}_{\mathrm{N}}$ (Supplementary Table S1; pCaMV35S::TaTypA-N ${ }_{1-80}:: G F P$ ). Wheat mesophyll protoplasts were then transformed by the polyethylene glycol (PEG) transfection method using the plasmid DNA of pCaMV35S::TaTypA::GFP and pCaMV35S::TaTypA$\mathrm{N}_{1-80}:: G F P, \quad$ CaMV35S::GFP as the control. The PEGtransfected mesophyll protoplasts were incubated in W5 solution in a dark chamber at $23^{\circ} \mathrm{C}$ for $18 \mathrm{~h}$, and GFP fluorescence was observed under a confocal laser scanning microscope (Zeiss LSM 700 , Germany) as previously described (Ito and Shinozaki, 2002).

The total protein in the protoplast pellet was extracted with protein extraction kits (Solarbio, Beijing, China) following the manufacturer's instructions. Western blotting assays were performed using the total protein by $12 \%$ SDS-PAGE. After the proteins were transferred to nitrocellulose membranes (Millipore), the membranes were incubated in blocking buffer (0.05\% Tween 20 and 5\% non-fat milk powder in TBS). GFPs were detected using mouse-derived GFP-antibodies (Sungene, Tianjing, China) diluted in blocking buffer at 1:5000 overnight at $4^{\circ} \mathrm{C}$. Membranes were washed and incubated with horseradish peroxidase-conjugated antimouse secondary antibody (Sungene, Tianjing, China) diluted at 1:10,000 and chemiluminescence substrate for detection (Sigma, Tokyo, Japan).

\section{BSMV-Mediated TaTypA Gene Silencing}

cDNA fragments of TaTypA with NotI and PacI restriction sites were obtained by reverse transcription PCR to modify the original BSMV: $\gamma$ vector for gene silencing as previously described (Holzberg et al., 2002). The fragments show no similarity with any other wheat gene in BLAST analyses, indicating their specificity. Capped in vitro transcripts of BSMV RNAs were prepared from the linearized plasmids (Petty et al., 1990) $\gamma$-TaPDS-as, $\gamma$-TaTypA-as, $\gamma, \alpha, \beta$ using a Message T7 in vitro transcription kit (Ambion, Austin, TX, USA) according to the manufacturer's protocol; $2.5 \mu \mathrm{l}$ of each transcript, including BSMV RNAs $\alpha, \beta$ and genetically modified $\gamma$, were added to $42.5 \mu \mathrm{l}$ of FES buffer $(0.1 \mathrm{M}$ glycine, $0.06 \mathrm{M} \mathrm{K}_{2} \mathrm{HPO}_{4}$, $1 \% \mathrm{w} / \mathrm{v}$ tetrasodium pyrophosphate, $1 \% \mathrm{w} / \mathrm{v}$ bentonite, and $1 \% \mathrm{w} / \mathrm{v}$ celite, $\mathrm{pH} 8.5$ ) and inoculated into the second leaves of two-leaf wheat seedlings by gentle rubbing of the surface with a gloved finger (Scofield et al., 2005). After $24 \mathrm{~h}$ of incubation in a dark, humid environment, the seedlings were maintained in a growth chamber at $25^{\circ} \mathrm{C}$ with a $16 \mathrm{~h}$ photoperiod, and virus symptoms were 
monitored at regular intervals. Recombinant virus BSMV:TaPDSas was used as a positive control in all experiments. Mocktreated plants were inoculated with $1 \times$ Fes buffer as a negative control. When virus symptoms were observed at 10 days post-viral inoculation, symptom phenotypes were photographed, and the fourth seedling leaves were inoculated with fresh urediniospores of Pst pathotypes CYR23 or CYR31. For RNA isolation and histological observation, leaves were sampled at 0, 24, 48, and 120 hpi with CYR23. The Pst infection phenotypes were also photographed at $15 \mathrm{dpi}$. This experiment was conducted with three independent biological replications.

\section{Histology of Fungal Growth and Host Response}

Wheat leaves infected with BSMV were sampled at 24, 48, and 120 hpi with Pst and stained as described (Wang et al., 2007). The fourth leaves pre-infected with BSMV were sampled at 24, 48, and 120 hpi with Pst race CYR23. The areas of necrosis in infected leaves were estimated by autofluorescence of mesophyll cells. Hyphal length and hyphal branches were examined under blue light excitation by epifluorescence microscopy (excitation filter, $485 \mathrm{~nm}$; dichromic mirror, $510 \mathrm{~nm}$; and barrier filter, $520 \mathrm{~nm}$ ). $\mathrm{H}_{2} \mathrm{O}_{2}$ accumulation was detected by staining with 3,3'-diaminobenzidine (DAB; Amresco, Solon, OH, USA) as previously described (Wang et al., 2007), then viewed by differential interference contrast optics. Only a site where an appressorium had formed over a stoma was considered to be successfully penetrated. At least 50 infection sites were examined on each of five randomly selected leaf segments for each treatment. Necrotic areas, areas of $\mathrm{H}_{2} \mathrm{O}_{2}$ accumulation, and hyphal length were observed with an Olympus BX-53 microscope (Olympus, Corp., Tokyo) and estimated with DP2-TWAIN/DP2-BSW software. Statistical analysis was performed by Tukey's HSD test $(P<0.05)$ with the use of SPSS software (SPSS, Inc., Chicago, IL, USA).

\section{RESULTS}

\section{Cloning and Structural Features of TaTypA}

A wheat 2,278-bp homolog of tyrosine phosphorylation protein A, first identified in silico, was obtained. It had an open reading frame (ORF) of 2,028 bp and was designated as TaTypA (GenBank accession number KF309066). Sequence alignment in the Chinese Spring genome database indicated that each subgenome $(\mathrm{A}, \mathrm{B}$, or $\mathrm{D})$ contained more than three sequences, each lacking the full TaTypA coding sequence likely due to incomplete Chinese Spring genome sequences. However, all sub-genomic sequences were localized on the long arm of chromosomes $6 \mathrm{~A}, 6 \mathrm{~B}$, and $6 \mathrm{D}$ (data not shown). To better understand $T a T y p A$ characteristics in the wheat genome, the exon of each sub-genomic sequence was selected and assembled artificially, designated TaTypA-6A, ТaTypA-6B, and TaTypA-6D, respectively (Supplementary Figure S1). The ТaTypA gene from wheat cv. Su11 exhibited 98.67, 77.78 and 98.52\% identities with the sequences from ТаТуpA-6A, ТаТ $y p A-6 B$, and ТаТypA$6 D$, respectively (Supplementary Figure S1). TaTypA-6A and TaTypA-6D contain only a few amino acid variations relative to TaTypA from Su11, whereas TaTypA-6B contains 101- and 48-aa deletions (Supplementary Figure S2). We concluded that the wheat genome contains three homologous copies of TaTypA located on the long arms of chromosomes $6 \mathrm{~A}, 6 \mathrm{~B}$, and 6D.

Sequence analysis indicated that TaTypA encodes a putative protein composed of 675 amino acid residues, a molecular weight of $74.25 \mathrm{kDa}$, and an isoeletric point (pI) of 5.65. Multi-sequence alignment with TypA from other higher plants showed that Su11 TaTypA shares highest identity (94.09\%) with TypA from Brachypodium distachyon. In addition, TaTypA shared 89, 75, and 74\% identity with OsTypA, SsTypA1, and AtTypA, respectively (Supplementary Figure S3). Structural analysis showed that TaTypA has three conserved regions, including an ATP/GTP-binding motif, a GTP-binding elongation factor signature, and a putative ribosome-binding domain (Supplementary Figure S3). Conserved tyrosine residues were also present.

TypA/BipA proteins from different organisms were selected to study the evolutionary relationships of TypA homologs. The neighbor-joining phylogenetic tree (Figure 1) showed that the proteins share a common ancestor with bacterial TypA and can be grouped into four groups. Plant TypA proteins formed a monophyletic group containing monocot and eudicot subgroups. TaTypA, together with OsTypA from Oryza sativa and BdTypA from $B$. distachyon, constituted a monocot subgroup. Homologous proteins from dicotyledonous plants clustered into another group. The short branch length (Figure 1) indicated that TypA proteins from different organisms share close evolutionary relationships.

\section{TaTypA Is Mainly Localized in Chloroplasts of Wheat Cells}

To determine the subcellular localization of TaTypA in wheat, recombinant pCaMV35S::TaTypA::GFP and pCaMV35S::TaTypA-N $1-80:: G F P$ were transfected into wheat mesophyll protoplasts by transfection. The empty pCaMV35S::GFP vector was used as a control. GFP was ubiquitously distributed throughout the cell, including in the nucleus (Figure 2). pCaMV35S::TaTypA::GFP and pCaMV35S::TaTypA-N $1-80::$ GFP fusion proteins were mainly targeted to the chloroplasts of wheat cells (Figure 2). To further confirm this result, a Western blot was performed using GFP antibodies on the subcellular fractions of pCaMV35S::GFP, pCaMV35S::TaTypA::GFP, and pCaMV35S::TaTypA-N $1-80::$ GFP transfected wheat protoplasts. Bands of 28-, 107-, and $35-\mathrm{kDa}$ were detected, suggesting that GFP, TapA-GFP and TaTypA-N $1-80-G F P$ was expressed in wheat cells (Supplementary Figure S4). These results from fluorescence microscope and Western blot analysis indicated that TaTypA-GFP was targeted to wheat chloroplast. 


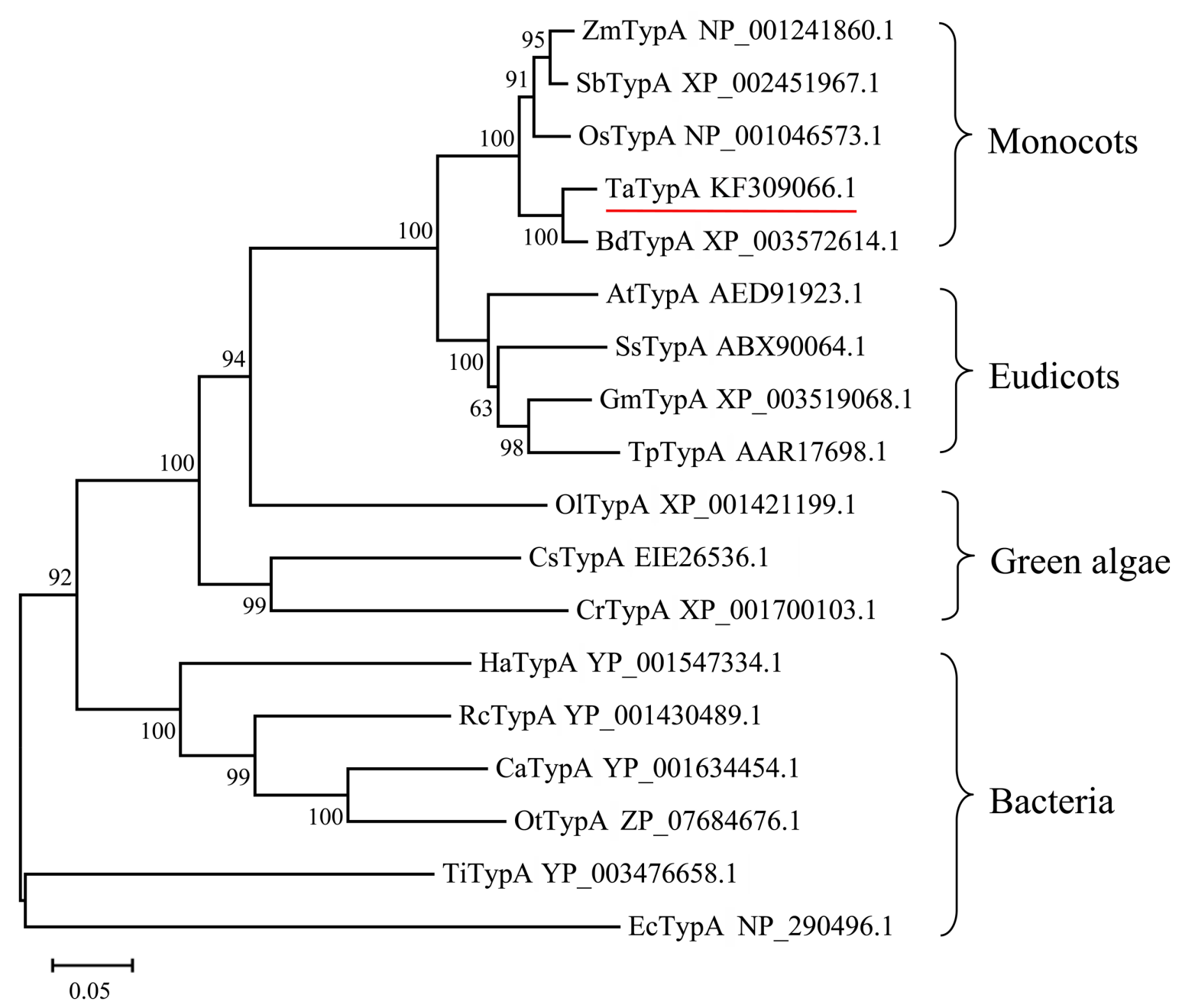

FIGURE 1 | Neighbor-joining tree of TypA protein sequences based on multiple alignments. The number above the internal branches indicates bootstrap values estimated based on 1,000 replications. Scale bar represents the estimated number of amino acid substitutions per site. Branches are labeled with protein names and GenBank accession numbers. Ta, Triticum aestivum; Bd, Brachypodium distachyon; Os, Oryza sativa; Zm, Zea mays; Sb, Sorghum bicolor; Gm, Glycine max; Ss, Suaeda salsa; At, Arabidopsis thaliana; Tp, Trifolium pretense; OI, Ostreococcus lucimarinus; Cs, Coccomyxa subellipsoidea; Cr, Chlamydomonas reinhardtii; Ca, Chloroflexus aurantiacus; Rc, Roseiflexus castenholzii; Ha, Herpetosiphon aurantiacus; Ot, Oscillochloris trichoides; Ti, Thermoanaerobacter italicus; Ec, Escherichia coli.

\section{Transcript Profiles of TaTypA in Response to Oxidative Stress and Abiotic Stress}

When treated with MV, a superoxide anion propagator, the wheat seedlings wilted due to oxidative stress (Supplementary Figure S5). Wheat seedlings treated with $5 \mathrm{mM}$ MV rapidly showed damping off symptoms, whereas seedlings treated with $0.1 \mathrm{mM}$ MV were only slightly affected up to 12 hpt. However, seedlings treated with $1 \mathrm{mM}$ MV expressed intermediate symptoms. Therefore, $1 \mathrm{mM} \mathrm{MV}$ was selected as a suitable concentration for the oxidative stress treatment. TaTypA transcripts were up-regulated and peaked as early as 4 hpt at a level approximately sixfold higher. The transcript level subsequently fell reaching the basal level at 6 and $8 \mathrm{hpt}$, but increased again at $12 \mathrm{hpt} \mathrm{until} 24 \mathrm{hpt}$ (Figure 3A).
Considering the involvement of TypA in response to various abiotic stresses in $S$. salsa, we investigated the effects of various abiotic stresses on expression of TaTypA. The transcript level of TaTypA increased threefold at $6 \mathrm{~h}$ after low-temperature treatment and reached a maximum of about sevenfold higher than the base level at $12 \mathrm{hpt}$ (Figure 3B). No significant change (less than twofold, $P>0.05$ ) in TaTypA transcript was detected in wheat leaves after treatment with salt or PEG (Figure 3B).

\section{Transcript Analysis of TaTypA in Response to Pst}

To investigate whether TaTypA is involved in response to Pst, quantitative RT-PCR was used to analyze TaTypA transcript profiles in both compatible and incompatible interactions. In the incompatible combination, transcript levels were induced at 36 


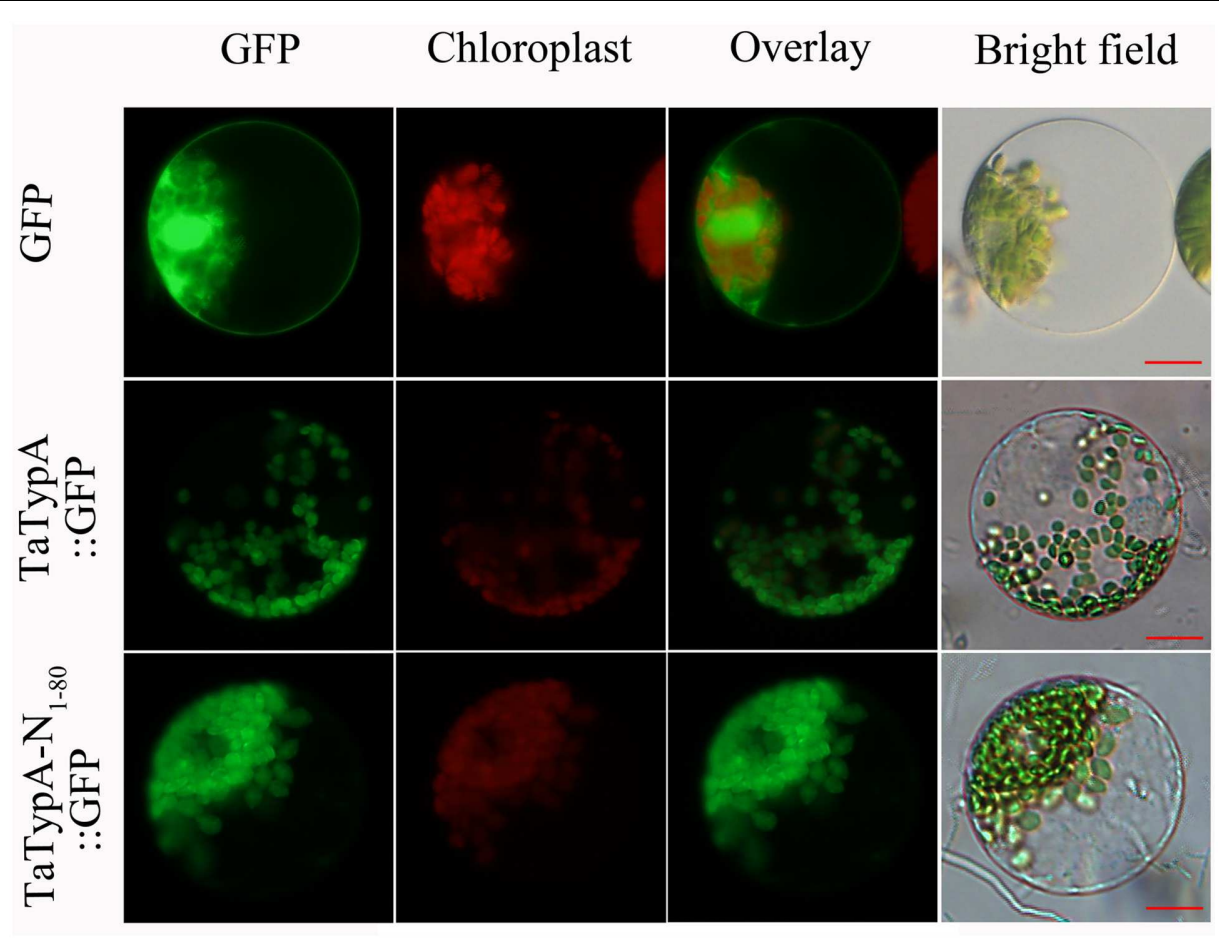

FIGURE 2 | Subcellular localization of TaTypA protein in wheat protoplasts. GFP signals are indicated by green color. All images were observed with a fluorescence microscope. Bar, $20 \mu \mathrm{m}$.

hpi and reached the highest level of 2.5-fold higher that in control plants at $96 \mathrm{hpi}$. In the compatible combination there was no significant change in relative TaTypA expression (Figure 4).

\section{Suppression of TaTypA Enhances Susceptibility of Wheat to Pst}

Virus-induced gene silencing (VIGS) is a rapid and effective reverse genetics approach in barley and wheat (Holzberg et al., 2002; Cakir and Scofield, 2008). To characterize in more detail the effect of the TaTypA gene in stripe rust response, BSMVmediated VIGS was employed to silence the TaTypA gene(s). Su11 seedlings inoculated with BSMV:TaPDS-as exhibited strong photobleaching symptoms at $10 \mathrm{dpi}$. The presence of non-symptomatic new leaves in plants treated with Fes buffer under the same conditions indicated that silencing of TaPDS occurred specifically in BSMV:TaPDS-as infected plants. All wheat seedlings inoculated with BSMV: $\gamma$ and BSMV:TypA-as displayed mild chlorotic mosaic symptoms on the third leaf at $10 \mathrm{dpi}$ (Figure $\mathbf{5 A}$ ), confirming that the BSMV-mediated gene silencing system functioned correctly and could be used in further experiments. Silencing efficiency assessed by qRT-PCR showed that TaTypA transcript levels were significantly reduced in TaTypA knock-down plants compared to control plants, with a reduction as high as $80 \%$ (Figure 5B). When fourth leaves of wheat plants were inoculated with the avirulent or virulent Pst races, an obvious HR was elicited by the avirulent CYR23 race on leaves pre-infected with BSMV: $\gamma$, BSMV:TaTypA-as and on mock-inoculated seedlings. Nevertheless, only limited fungal sporulation had occurred around the necrotic spots on leaves pre-infected with BSMV:TaTypA-as after $15 \mathrm{dpi}$ compared to the other two treatments (Figure 5C). By contrast, wheat seedlings inoculated with virulent CYR31 showed normal disease development and numerous uredinia (Figure 5D). The specific fragment used in silencing is shown in Supplementary Figure S1. However, due to the high identity and similarity among the three copies, the silencing efficiency of each copy on A, B, and D could not be determined.

\section{Pst Growth and Host Response}

Leaf segments from at least three plants inoculated with CYR23 were harvested from each sample to examine detailed histological changes associated with the enhanced susceptibility in TaTypA knock-down plants. Areas of cell death were obvious at 24, 48, and 120 hpi by microscopy and were estimated by DP-BSW software (Figure 6A). The areas were significantly less in TaTypA knock-down plants than in BSMV: $\gamma$-infected plants at 48 and 120 hpi (Figure 6B). There was no obvious difference in hyphal growth and branching at 24 hpi between TaTypA knock-down and BSMV: $\gamma$ plants (Figures 6C,D). However, hyphal length and branch number in BSMV:TaTypA-as infected leaves were significantly $(P<0.05)$ increased relative to those in BSMV: $\gamma$ infected leaves at 48 and 120 hpi, respectively (Figures 6C,D). The collective histological results suggest that silencing of TaTypA weakened 
A

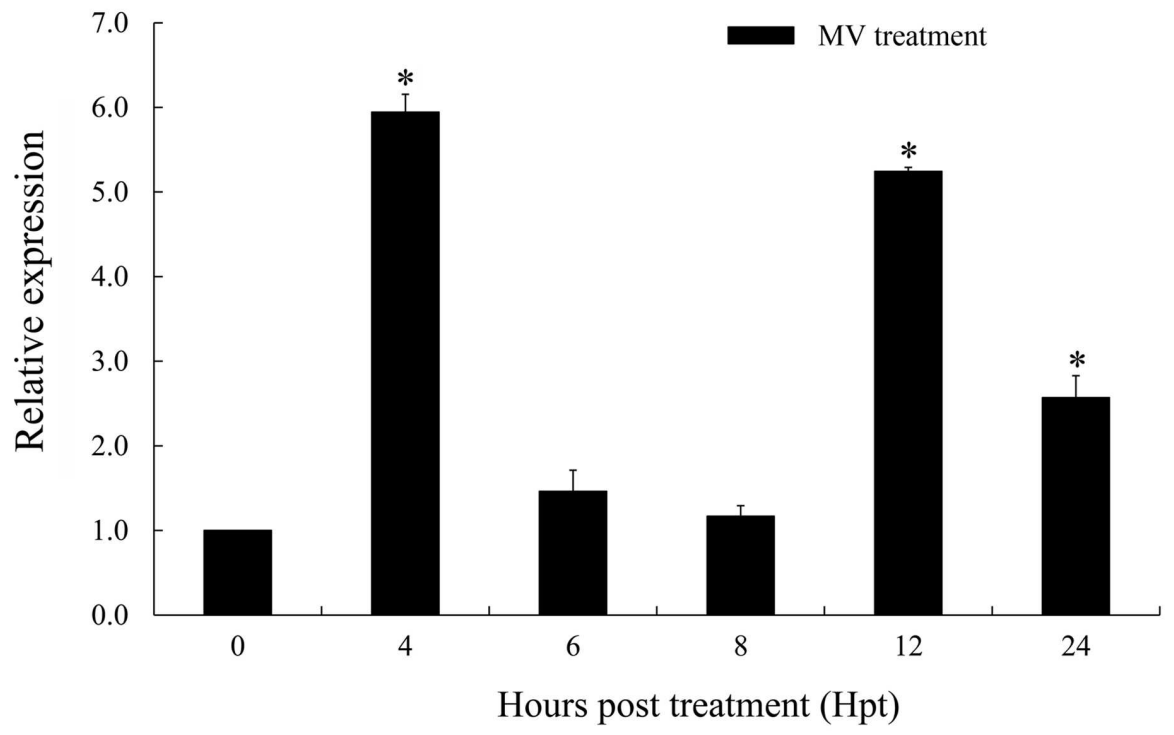

B

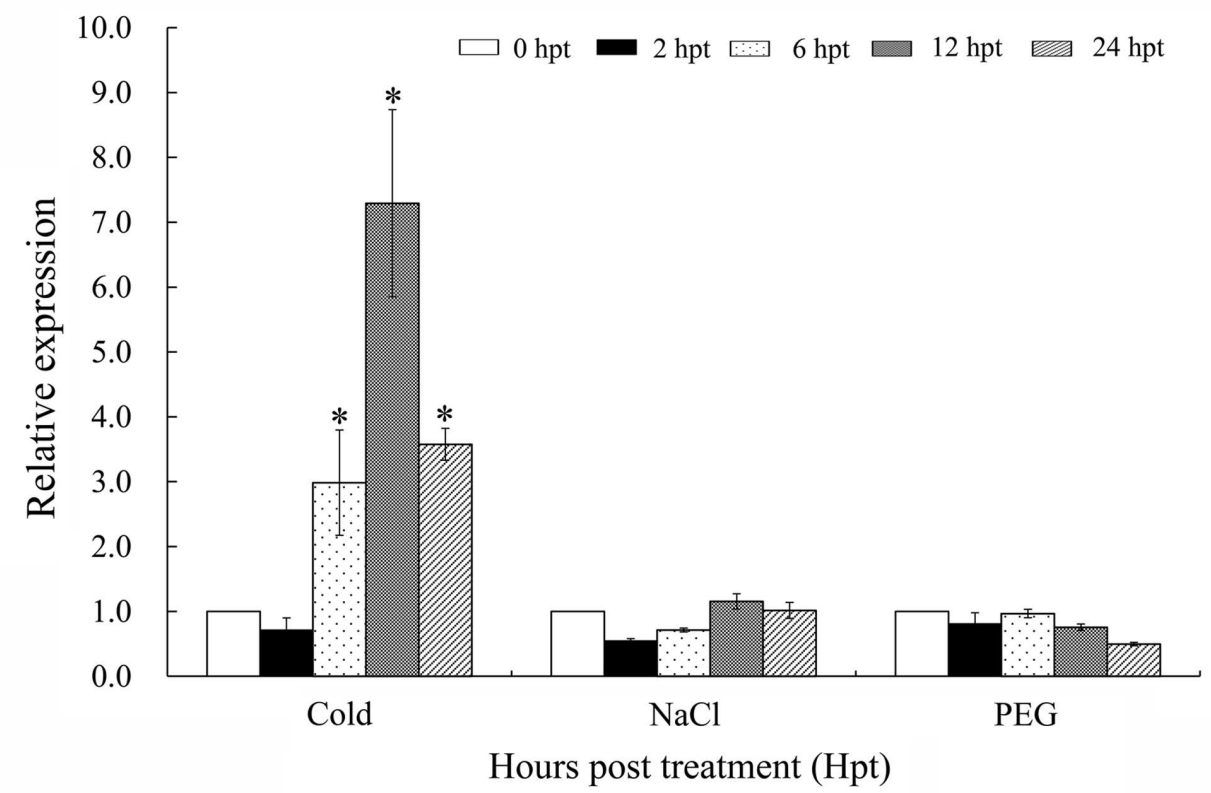

FIGURE 3 | Expression patterns of TaTypA in wheat leaves based on qRT-PCR analyses. (A) Expression levels of TaTypA in wheat leaves in response to MV treatment. (B) Expression profiles of TaTypA in wheat leaves after treatment with environmental stresses: low temperature ( $\left.4^{\circ} \mathrm{C}\right)$, salt (NaCl), and drought (PEG). The expression levels were analyzed by qRT-PCR and normalized to the wheat elongation factor TaEF-1a gene. Means and standard deviations were based on three independent biological replicates. Asterisks indicate a significant difference $(P<0.05)$ from 0 hpt by Tukey's HSD test.

the resistance of in Su11 and permitted enhanced hyphal growth and branching. The transcription level of SA-induced marker gene TaPR1 was significantly reduced in CYR23infected TaTypA knock-down plants at 48 and $120 \mathrm{hpi}$, indicating a concurrent effect on defense-related gene expression (Figure 6E).

\section{Weakened ROS Accumulation in TaTypA}

\section{Knock-down Plants}

Reactive oxygen species accumulation with the occurrence of $\mathrm{HR}$ occurs in the resistance response to rust fungi in wheat (Wang et al., 2007). The induction of $\mathrm{H}_{2} \mathrm{O}_{2}$ by infection in TaTypA knock-down plants was analyzed by histochemical 


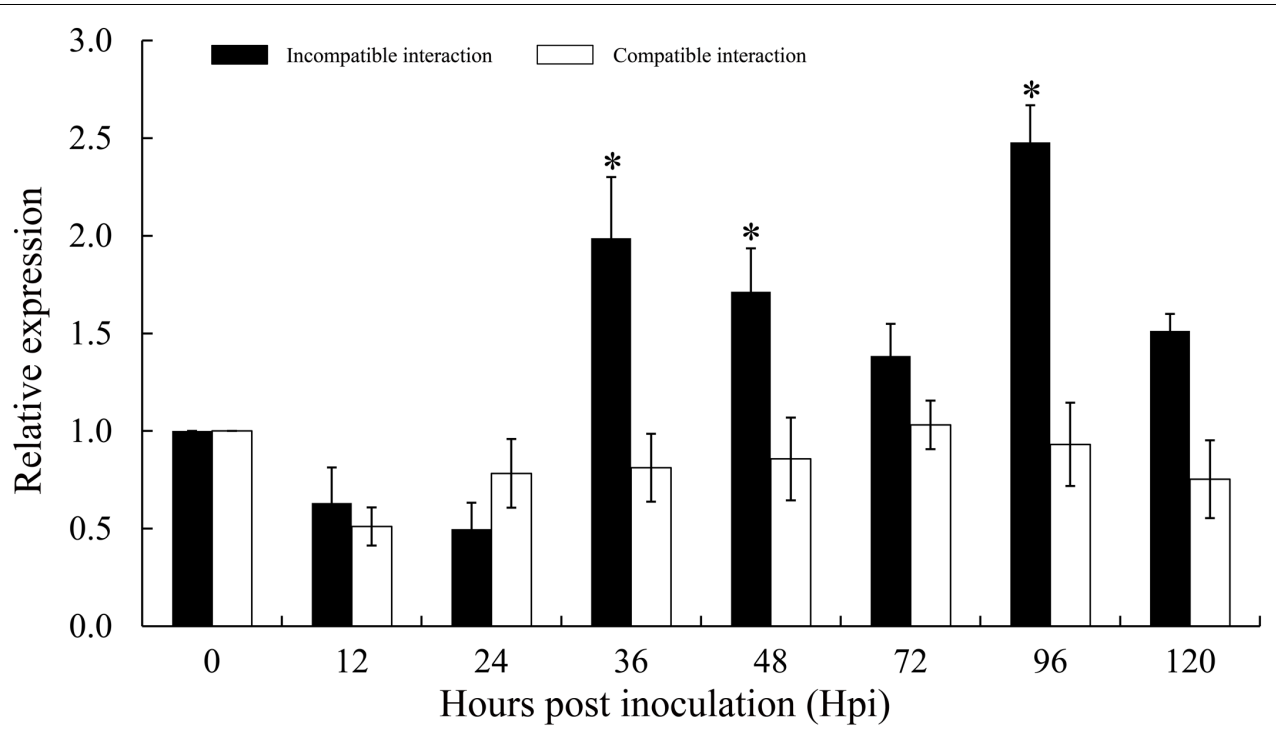

FIGURE 4 | Expression profiles of TaTypA in wheat leaves in response to virulent and avirulent Pst races. Wheat leaves inoculated with Pst isolates CYR23 (avirulent) and CYR31 (virulent) were sampled at 0, 6, 12, 24, 48, and $120 \mathrm{~h}$ post-inoculation. The data were normalized to the wheat TaEF-1a gene, and error bars represent the variation among three independent replicates. Asterisks indicate significant differences from 0 hpi using Tukey's HSD test $(P<0.05)$.

observation and colorimetric determination. $\mathrm{H}_{2} \mathrm{O}_{2}$ accumulation was significantly decreased in TaTypA knock-down plants compared to BSMV: $\gamma$ infected plants at 48 and $120 \mathrm{hpi}$ (Figures 7A,B). In addition, expression levels of the TaCAT and TaSOD genes involved in ROS-scavenging were significantly upregulated in TaTypA knock down plants infected with CYR23 (Figure 7C).

\section{DISCUSSION}

TypA proteins are a novel class of stress tolerance-related proteins in all species ranging from prokaryotes to higher eukaryotes (Wang et al., 2008). In this study, we conducted molecular and functional analyses of wheat TaTypA in a wheatPst pathosystem.

As many as $87 \%$ of the genes in hexaploid wheat are triplicated across the A, B, and D sub-genomes (Pumphrey et al., 2009). Therefore, not unexpectedly there were three copies of TaTypA in the wheat genome. However, sequence alignment of three genomic copies of TaTypA showed that TaТypA-6A, ТаТурA-6B and TaTypA-6D differed by sequence variation and deletion (Supplementary Figure S1). As described in previous reports, newly formed polyploids undergo a speciation process driven by combined effects of chromosomal re-patterning, gene deletion, mutation, suppression (silencing), or acquisition of modified gene function (Wendel, 2000). Thus, the differences in the three genomic copies of TaTypA are consistent with such events. Sequence analysis revealed that TaTypA is a typical member of the ribosome-binding GTPase superfamily, which is characterized by a ATP/GTPbinding motif, a GTP-binding elongation factor signature, and a putative ribosome-binding domain (Kiss et al., 2004).
Phylogenetic analysis indicated that TypA proteins in plants form a monophyletic group, within which TaTypA and rice OsTypA constitute a subgroup (Figure 1). The presence of different subgroups within and between monocots and eudicots indicate possibilities for functional divergence between species and, in the case of allopolyploid plants, between subgenomes.

In $S$. salsa, the SsTypA1-GFP fusion protein localized in the chloroplasts, and structural analysis revealed a chloroplast transit peptide sequence at the N-terminal part of the SsTypA1 protein (Wang et al., 2008). Without a chloroplast transit peptide the chloroplast localization of the protein was unexpected (Shi and Theg, 2013). In this study, we proved that the $\mathrm{N}$-terminal region and the full length of TaTypA have the same subcellular localization as SsTypA1, indicating that the $\mathrm{N}$-terminal region of TaTypA does contain a chloroplast transit peptide sequence. It has been confirmed that chloroplasts play a significant role in defense responses (de Torres Zabala et al., 2015). The chloroplast is a major source of ROS in plant cells and is responsible for producing the two signaling molecules required for pathogen responses, SA and jasmonic acid (Almagro et al., 2009). In addition, a few reports have shown that the resistance proteins are targeted to this organelle. For example, wheat stripe rust resistance protein WKS1 localized in chloroplasts and reduced the ability of the thylakoid-associated ascorbate peroxidase to detoxify ROS (Gou et al., 2015), and the chloroplastic/cytoplasmic protein NRIP1, which associated with the NB-LRR immune receptor "N", was shown to be responsible for tobacco mosaic virus recognition and resistance in Nicotiana (Caplan et al., 2008). In this study, ROS accumulation was reduced during Pst infection in TaTypA-knock down plants. Our data suggest that TaTypA is a new chloroplast-localized defense protein, which is involved in wheat resistance to Pst. 
A

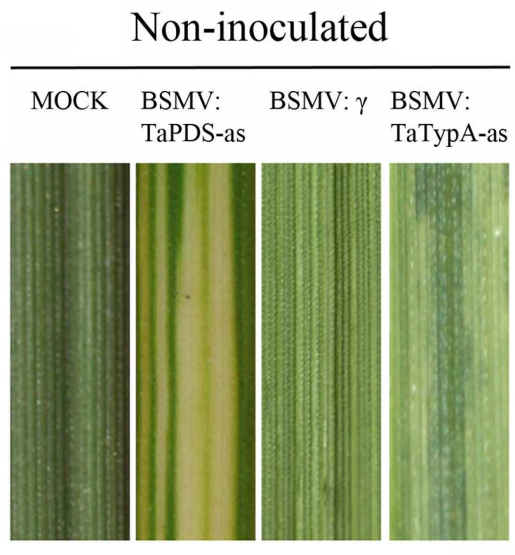

C

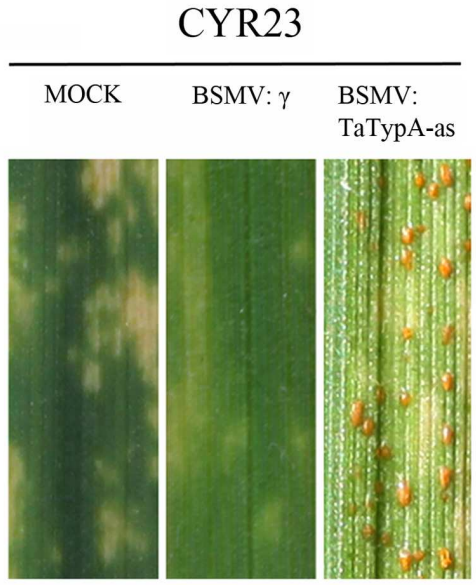

B

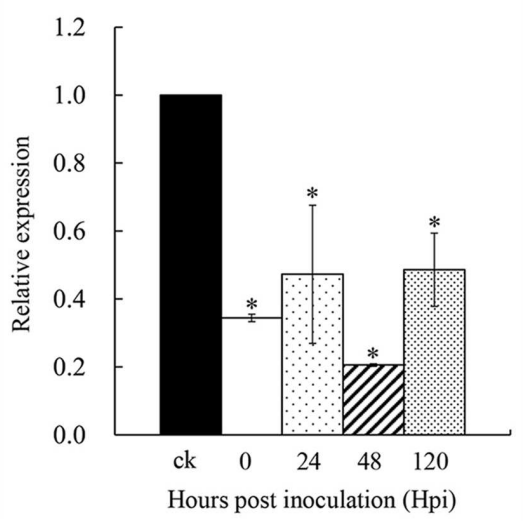

D

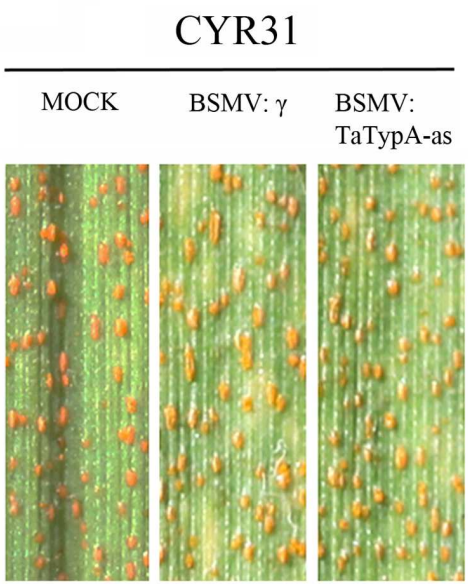

FIGURE 5 | Functional characterization of the TaTypA gene during interaction of wheat and Pst by BSMV-mediated gene silencing. (A) Mild chlorotic mosaic symptoms on the fourth leaves of seedlings inoculated with BSMV: $\gamma$, BSMV:TaPDS-as, and BSMV:TaTypA-as at 10 dpi. Mock: wheat leaves treated with $1 \times$ Fes buffer. (B) Relative transcript levels of TaTypA in TaTypA knock-down plants inoculated with CYR23. The data were normalized to the TaEF-1a gene. CK indicates BSMV: $\gamma$. Asterisks indicate significant differences from BSMV: $\gamma$ using Tukey's HSD test $(P<0.05)$. Photographs of fourth leaves further inoculated with urediniospores of avirulent race CYR23 (C) or virulent race CYR31 (D). Typical leaves were photographed at 15 dpi.

Reactive oxygen species can be produced in chloroplasts through photoreaction of the herbicide MV, a superoxide anion propagator (Scarpeci et al., 2008). Wheat not only wilted when treated with certain concentrations of MV (Supplementary Figure S4), but transcript levels of TaTypA were significantly elevated in wheat seedlings exposed to MV (Figure 3A). This response is similar to that of $S s T y p A$, which showed pronounced activation in response to $\mathrm{H}_{2} \mathrm{O}_{2}$ treatment (Wang et al., 2008). ROS, such as superoxide, hydrogen peroxide, and hydroxyl radicals, were induced by drought, salt, and cold stress. The increased expression of TaTypA in response to cold stress (Figure 3B) may result from the excessive ROS generated. However, the transcript level of TaTypA was not significantly changed under salt or drought stress. As previous studies showed, the biphasic production of ROS consists of a primary phase that occurs within minutes and a secondary phase that occurs within hours/days under abiotic stress (Mittler et al., 2011). It seems that TaTypA was not involved in primary phase of ROS production under drought and cold stress. On the contrary, SsTypA1 was induced by $\mathrm{NaCl}$ and mannitol (drought stress) treatments as early as 6 and $4 \mathrm{hpt}$, respectively (Wang et al., 2008). These differences in expression of TaTypA and $S s T y p A 1$ may be associated with divergence of monocots and dicots or different treatment methods used. Different plant species are highly variable in response to environmental factors; an environmental condition that is harmful to one species may not be stressful for another (Munns and Tester, 2008). This is also evidenced by the multitude of different stress-response mechanisms. Hence, we propose that the functions of TaTypA and SsTypA1 differ when plants are subjected to the same environmental stresses.

TypA/BipA proteins are widely distributed in all organisms. However, there has been little research on the functions of TypA proteins in plant disease responses. Our results in the present study indicated that TaTypA positively regulates 


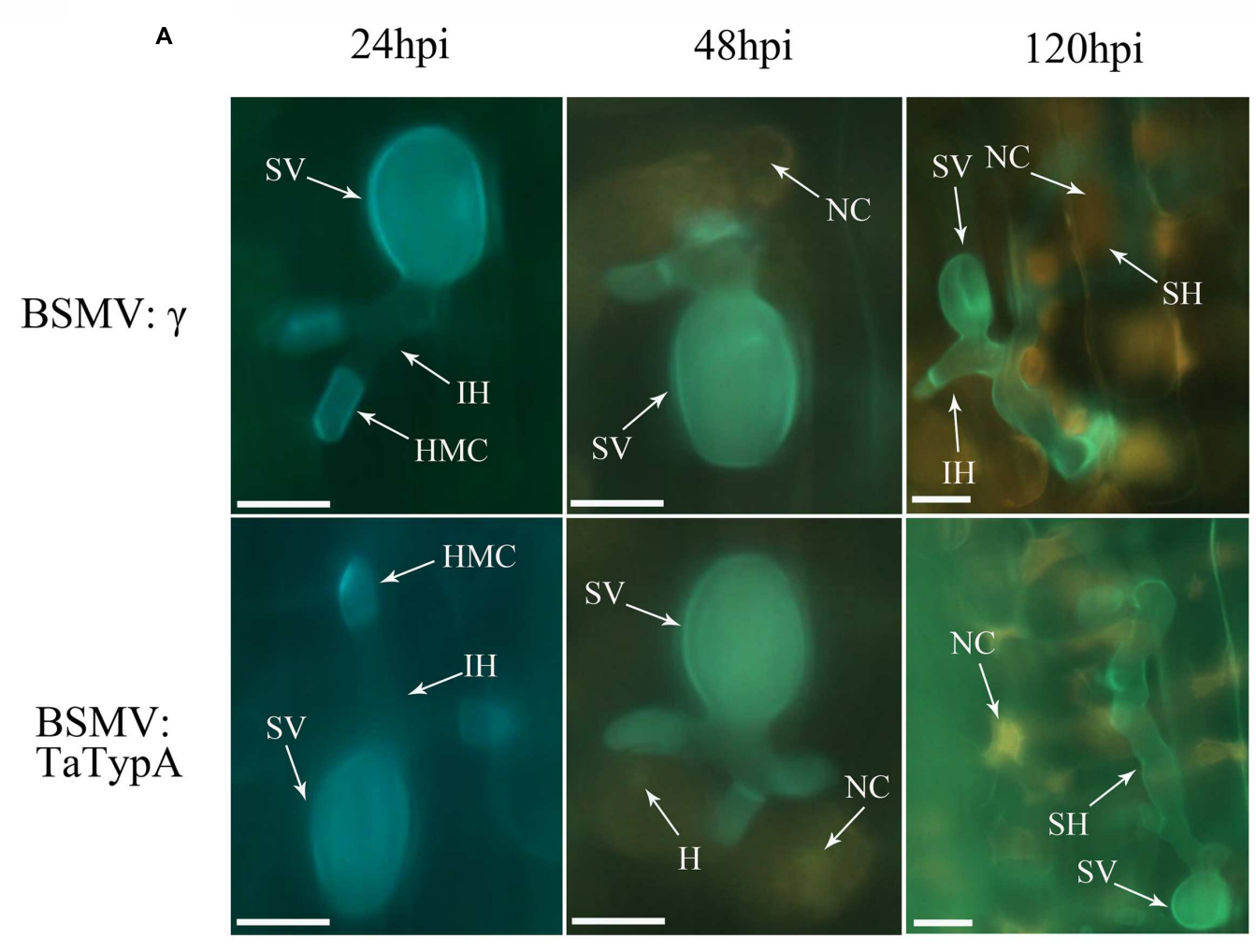

B

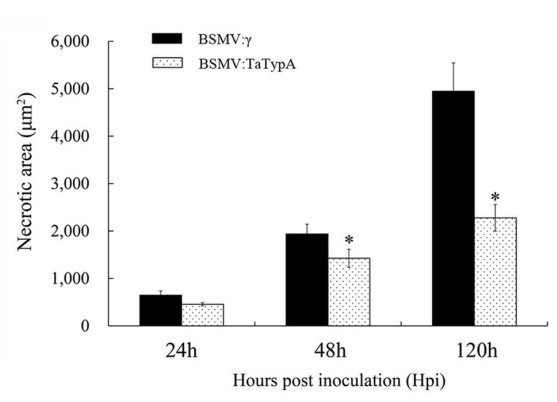

D

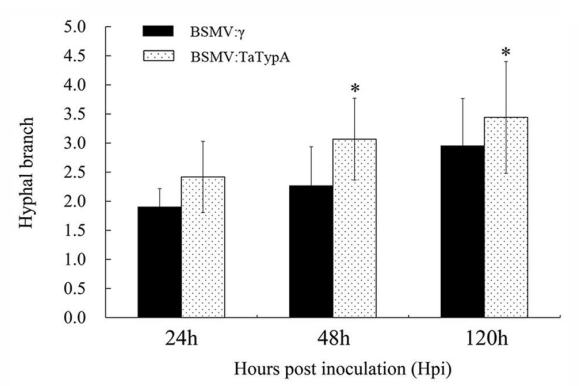

C

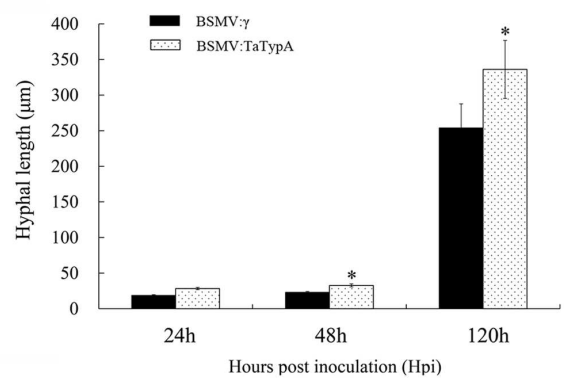

E

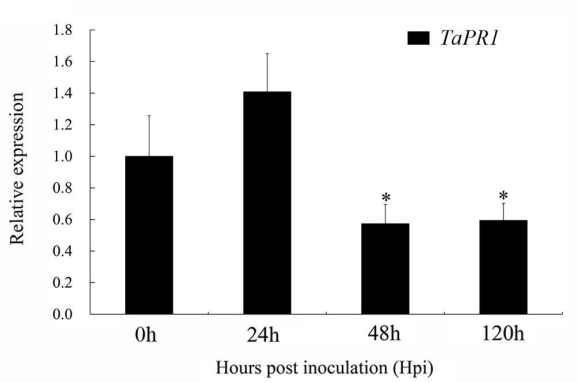

FIGURE 6 | Weakened wheat defense response in TaTypA knock-down plants challenged by avirulent Pst race CYR23. Wheat leaves previously treated with recombinant BSMV: $\gamma$ or BSMV:TaTypA were inoculated with race CYR23. (A) Typical leaves were examined at 24, 48 and 120 hpi. The infection sites were observed by epifluorescence. HMC, haustorial mother cell; SV, substomatal vesicle; NC, necrotic cell death; IH, primary hyphae; SH, secondary hyphae. All results were obtained from 50 infection sites. Bar, $20 \mu \mathrm{m}$. (B) Necrotic cell death was quantified as the area of autofluorescence. (C) Hyphal lengths are means of the average distance from the junction of the substomatal vesicle to the hyphal tip. (D) Hyphal branch values are means of average numbers of primary hyphae.

(E) Expression of TaPR1 was assayed in TaTypA knock-down plants compared to controls. The data were normalized to the wheat TaEF-1a gene. Asterisks indicate a significant differences $(P<0.05)$ from BSMV: $\gamma$ using the Tukey's HSD test. 


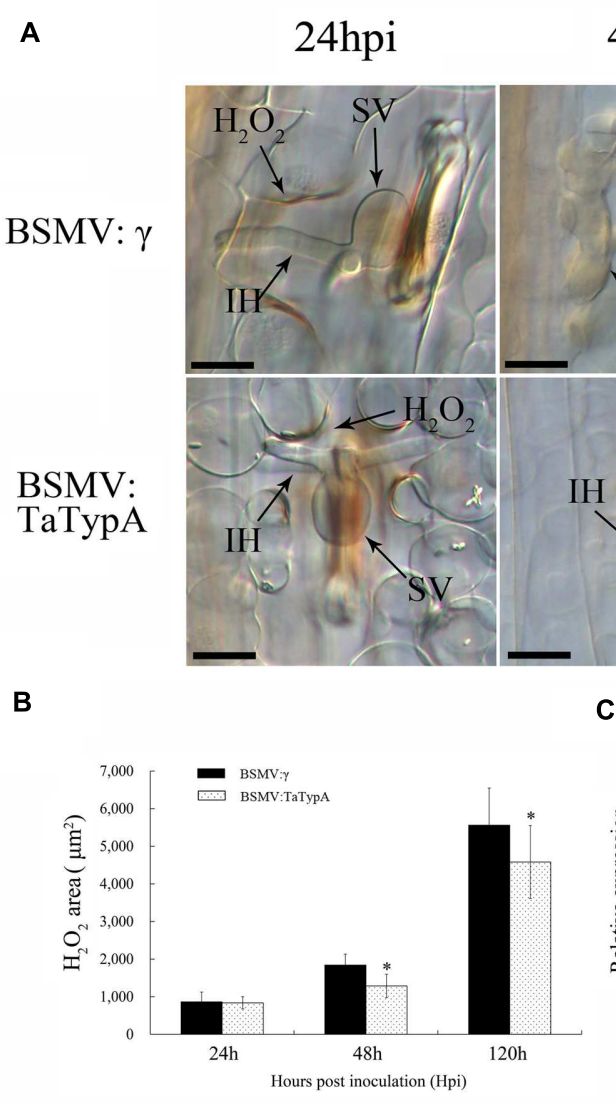

48hpi

$120 \mathrm{hpi}$
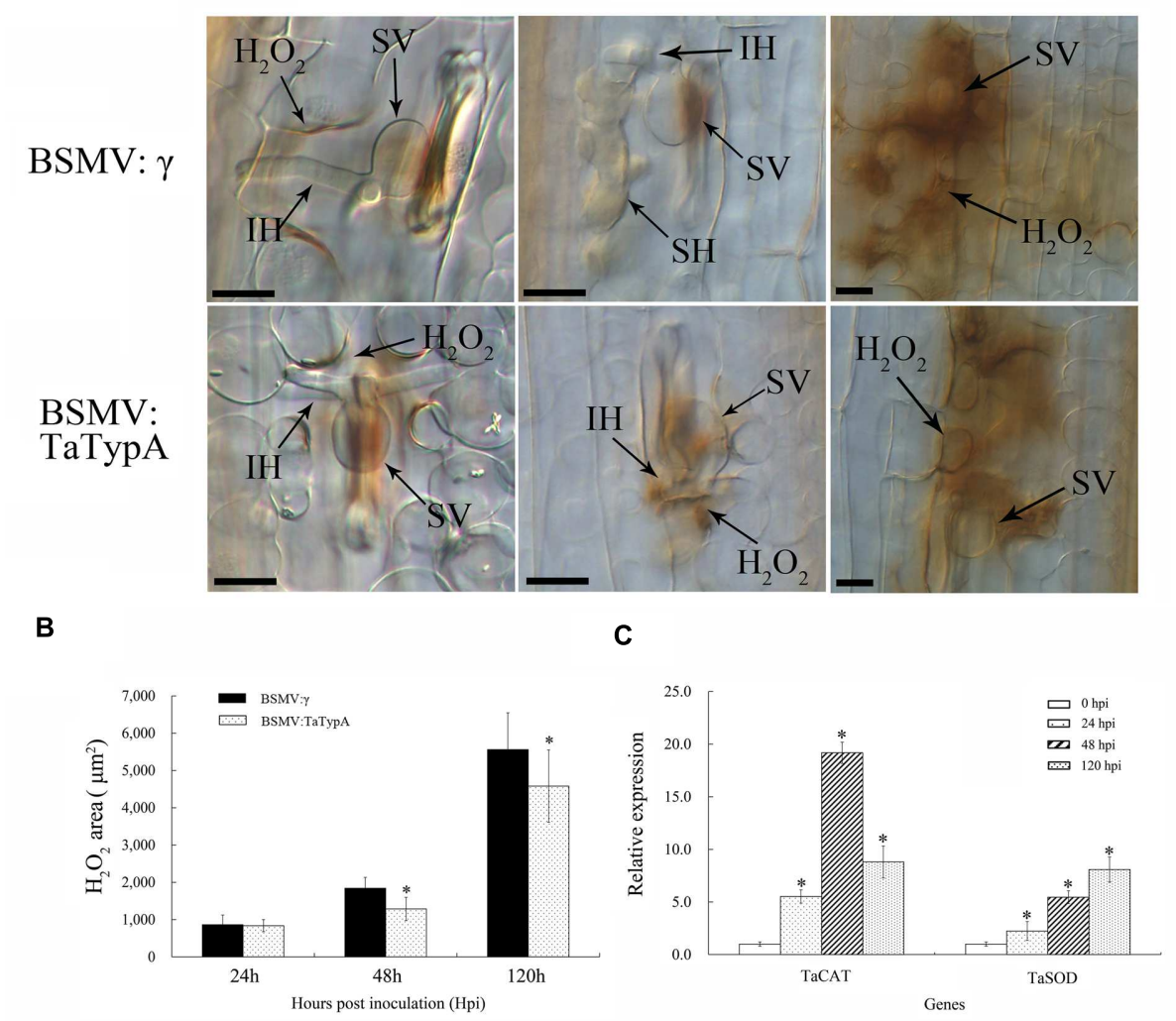

FIGURE 7 | Reduced $\mathrm{H}_{2} \mathrm{O}_{2}$ accumulation in TaTypA knock-down plants challenged by avirulent race CYR23. (A) Wheat leaves previously treated with recombinant BSMV: $\gamma$ or BSMV:TaTypA were inoculated with Pst race CYR23. $\mathrm{H}_{2} \mathrm{O}_{2}$ accumulation at infection sites was detected by staining with 3,3'-diaminobenzidine (DAB). Infection sites were observed by light microscopy. SV, substomatal vesicle; IH, initial hyphae; SH, secondary hyphae. Bar, $20 \mu \mathrm{m}$. (B) The amount of $\mathrm{H}_{2} \mathrm{O}_{2}$ production was measured by calculating the DAB-stained area at each infection site using DP-BSW software. Values represent mean \pm standard errors of three independent assays. (C) Expressions of two ROS-scavenging enzyme genes were assayed in TaTypA knock-down plants compared to control plants. The data were normalized to wheat the TaEF-1a gene. Asterisks indicate significant difference $(P<0.05)$ from BSMV: $\gamma$ using Tukey's HSD test.

the response of wheat to Pst. Silencing of TaTypA reduced the level of race-specific immunity to Pst in TaTypA knockdown plants. In response to the avirulent Pst race CY23, TaTypA knock-down Sul1 wheat plants expressed weakened resistance characterized by decreased necrotic area, reduced ROS accumulation and increased uredinia production. Previous histological and cytological observations revealed oxidative bursts during the early $(12-24 \mathrm{~h})$ and late $(96-120 \mathrm{~h})$ stages of infection in incompatible interactions between wheat and Pst (Wang et al., 2007). In contrast, the hyphae of Pst rapidly colonized host tissues during the compatible interaction without any detectable $\mathrm{O}_{2}{ }^{--}$and $\mathrm{H}_{2} \mathrm{O}_{2}$ accumulation or HR. TaTypA expression was induced as early as $36 \mathrm{hpi}$ and peaked at $96 \mathrm{hpi}$ in the incompatible interaction, suggesting that TaTypA may be responsible for ROS accumulation and protection of host cells from Pst infection. Thus, silencing of TaTypA led to decreased ROS accumulation in TaTypA-knock-down plants. Superoxide dismutase (SOD) and catalase (CAT) are major ROS-scavenging enzymes (Mittler et al., 2004). $\mathrm{H}_{2} \mathrm{O}_{2}$ can be eliminated by increased catalase and superoxidase activities. The accumulation of TaSOD and TaCAT in TaTypA knock-down plants was induced by CYR23 infection, suggesting that TaTypA is an important factor in the ROS-scavenging system. Decreased catalase and ascorbate peroxidase activities in tobacco resulted in plants with increased resistance to pathogens (Mittler et al., 1999), whereas overexpression of catalase resulted in plants that were more susceptible (Polidoros et al., 2001). These results suggest that the ROS-scavenging systems have an important role in managing ROS generated in response to pathogens. Our results suggest that TaTypA positively regulates resistance to stripe rust in a ROS-dependent manner.

Hyphal length and number of branches are measures the ability of the pathogen to colonize the host under the stress imposed by HR at infection sites. The necrotic area triggered by infection of avirulent race CYR23 was used to represent the level of the HR, which is probably triggered by generation of ROS (Wang et al., 2007; Zurbriggen et al., 2010). In TaTypA knock-down plants, ROS accumulation was reduced in CYR23 infection at 48 and $120 \mathrm{hpi}$, coinciding with decreased hyphal length, necrotic area and hyphal branch number, suggesting that 
increased colonizing ability of the pathogen is a consequence of decreased host resistance. ROS are the well-characterized second messengers in a variety of cellular processes in plants, including tolerance to environmental stress (Desikan et al., 2001). ROS has been shown to regulate different plant hormone signaling pathways, plant-biotic interactions and developmental processes by redox-dependent regulation of transcription factors (Barna et al., 2012). One of the most thoroughly characterized defense-signaling pathways regulated by oxidation events is the induction of salicylic acid (SA)dependent responses (Fu and Dong, 2013). In the present study, TaPR1, the marker gene of the SA pathway, was significantly decreased by CYR23 infection at 48 and 120 hpi. We infer that TaTypA, functions in the SA-signaling pathway via ROSdependent signals and thereby contributes to systemic acquired resistance.

To our knowledge this is the first report to confirm that TypA functions in biotic stress. Knock-down of TaTypA demonstrated its positive role in regulation of hypersensitive cell death and in resistance of wheat to stripe rust. On the basis of our results, we believe that TypA has an important role in plant disease resistance, but determination of the precise functional mechanism of TaTypA requires further investigation.

\section{AUTHOR CONTRIBUTIONS}

JG and ZK designed the experiment. PL, TM, WM, DL, TQ, and JG performed the experiments and analyzed the data. PL, TM, JG, and $\mathrm{ZK}$ wrote the manuscript.

\section{FUNDING}

This study was financially supported by National Basic Research Program of China (No. 2013CB127700), National Natural Science Foundation of China (No. 31371889 and 31171795), the Program for New Century Excellent Talents in University (NCET-12-0471), the 111 Project from the Ministry of Education of China (No. B07049), the Natural

\section{REFERENCES}

Almagro, L., Gómez Ros, L. V., Belchi-Navarro, S., Bru, R., Ros Barceló, A., and Pedreño, M. A. (2009). Class III peroxidases in plant defence reactions. J. Exp. Bot. 60, 377-390. doi: 10.1093/jxb/ern277

Atkinson, G. C. (2015). The evolutionary and functional diversity of classical and lesser-known cytoplasmic and organellar translational GTPases across the tree of life. BMC Genomics 16:1. doi: 10.1186/s12864-0151289-7

Barak, M., and Trebitsh, T. (2007). A developmentally regulated GTP binding tyrosine phosphorylated protein A-like cDNA in cucumber (Cucumis sativus L.). Plant Mol. Biol. 65, 829-837. doi: 10.1007/s11103-007-9246-8

Barna, B., Fodor, J., Harrach, B., Pogány, M., and Király, Z. (2012). The Janus face of reactive oxygen species in resistance and susceptibility of plants to necrotrophic and biotrophic pathogens. Plant Physiol. Biochem. 59, 37-43. doi: 10.1016/j.plaphy.2012.01.014
Science Foundation of Shaanxi Province (2014JM3059) and the Fundamental Research Funds for the Central Universities of China (YQ2013001).

\section{ACKNOWLEDGMENTS}

We thank Professor Larry Dunkle from the USDA-Agricultural Research Service at Purdue University, USA and Professor Robert Mcintosh from University of Sydney, Australia for critical reading of the manuscript.

\section{SUPPLEMENTARY MATERIAL}

The Supplementary Material for this article can be found online at: http://journal.frontiersin.org/article/10.3389/fpls.2016.00873

FIGURE S1 | Multi-alignment of the encoding sequence of the three copies of TaTypA. TaTypA cDNA, the full-length cDNA sequence amplified from cDNA of wheat cv. Suwon 11. The cDNA sequences of three TaTypA copies from chromosomes $6 \mathrm{AL}, 6 \mathrm{BL}$, and $6 \mathrm{DL}$ were obtained from the wheat UGRI genome database of wheat cv Chinese Spring. The red and blue boxes indicate the initiation codon (ATG) and termination codon (TAG), respectively.

FIGURE S2 | Multi-alignment of the deduced TaTypA proteins from wheat cv. Suwon11 and Chinese Spring genome database. Identical and similar amino acid residues are shaded in black and light gray, respectively.

FIGURE S3 | Multi-sequence alignment of TaTypA against TypA from other species. Identical and similar amino acid residues are shaded in black and light gray, respectively. Motifs indicated in the sequence: (I) ATP/GTP binding site; (II) GTP-binding elongation factor signature; (III) putative ribosome binding domain. Triangles indicate conserved tyrosine residues in all nine proteins. Ta, Triticum aestivum; Bd, Brachypodium distachyon; Os, Oryza sativa; Zm, Zea mays; Sb, Sorghum bicolor; Gm, Glycine max; Ss, Suaeda salsa; At, Arabidopsis thaliana; Tp, Trifolium pretense.

FIGURE S4 | The expression of GFP, TaTypA::GFP and TaTypA-N $\mathbf{N}_{1-80}:$ GFP proteins was determined by Western blot analysis using GFP antibody. The GFP antibody was diluted in blocking buffer at 1:5000. The graph shows the relative band using image Lab 3.0 software.

FIGURE S5 | Symptoms of wheat seedlings treated with MV. Ten-days-old wheat seedlings were sprayed with $0.1,1$, and $5 \mathrm{mM} M V$ under the normal growing conditions. CK, wheat seedlings sprayed with water.

TABLE S1 | Primers used for TaTypA analyses.

Cakir, C., and Scofield, S. (2008). Evaluating the ability of the barley stripe mosaic virus-induced gene silencing system to simultaneously silence two wheat genes. Cereal Res. Commun. 36, 217-222. doi: 10.1556/CRC.36.2008. Suppl.B.18

Cao, Z., Jing, J., Wang, M., Shang, H., and Li, Z. (2002). Relation analysis of stripe rust resistance gene in wheat important cultivar Suwon 11, Suwon 92 and Hybrid 46. Acta Bot. Boreal-Occident Sin. 23, 64-68.

Caplan, J. L., Mamillapalli, P., Burch-Smith, T. M., Czymmek, K., and Dinesh-Kumar, S. P. (2008). Chloroplastic protein NRIP1 mediates innate immune receptor recognition of a viral effector. Cell 132, 449-462. doi: 10.1016/j.cell.2007.12.031

Chenna, R., Sugawara, H., Koike, T., Lopez, R., Gibson, T. J., Higgins, D. G., et al. (2003). Multiple sequence alignment with the clustal series of programs. Nucleic Acids Res. 31, 3497-3500. doi: 10.1093/nar/gkg500

de Torres Zabala, M., Littlejohn, G., Jayaraman, S., Studholme, D., Bailey, T., Lawson, T., et al. (2015). Chloroplasts play a central role in plant 
defence and are targeted by pathogen effectors. Nat. Plants 1:15074. doi: 10.1038/nplants.2015.74

De Tullio, M. C. (2010). Antioxidants and redox regulation: changing notions in a changing world. Plant Physiol. Biochem. 48, 289-291. doi: 10.1016/j.plaphy.2010.02.011

Desikan, R., Soheila, A. H., Hancock, J. T., and Neill, S. J. (2001). Regulation of the Arabidopsis transcriptome by oxidative stress. Plant Physiol. 127, 159-172. doi: 10.1104/pp.127.1.159

Farris, M., Grant, A., Richardson, T. B., and O'Connor, C. D. (1998). BipA: a tyrosine-phosphorylated GTPase that mediates interactions between enteropathogenic Escherichia coli (EPEC) and epithelial cells. Mol. Microbiol. 28, 265-279. doi: 10.1046/j.1365-2958.1998.00793.x

Fu, Z. Q., and Dong, X. (2013). Systemic acquired resistance: turning local infection into global defense. Annu. Rev. Plant Biol. 64, 839-863. doi: 10.1146/annurevarplant-042811-105606

Gou, J. Y., Li, K., Wu, K., Wang, X., Lin, H., Cantu, D., et al. (2015). Wheat stripe rust resistance protein WKS1 reduces the ability of the thylakoid-associated ascorbate peroxidase to detoxify reactive oxygen species. Plant Cell 27, 17551770. doi: $10.1105 /$ tpc. 114.134296

Grant, A. J., Farris, M., Alefounder, P., Williams, P. H., Woodward, M. J., and O'Connor, C. D. (2003). Co-ordination of pathogenicity island expression by the BipAGTPase in enteropathogenic Escherichia coli (EPEC). Mol. Microbiol. 48, 507-521. doi: 10.1046/j.1365-2958.2003.t01-1-03447.x

Hau, B., and de Vallavieille-Pope, C. (2006). "Wind-dispersed diseases," in The Epidemiology of Plant Diseases, ed. B. M. Cooke (Dordrecht: Springer), 387-416.

Holzberg, S., Brosio, P., Gross, C., and Pogue, G. P. (2002). Barley stripe mosaic virus-induced gene silencing in a monocot plant. Plant J. 30, 315-327. doi: 10.1046/j.1365-313X.2002.01291.x

Ito, T., and Shinozaki, K. (2002). The MALE STERILITY1 gene of Arabidopsis, encoding a nuclear protein with a PHD-finger motif, is expressed in tapetal cells and is required for pollen maturation. Plant Cell Physiol. 43, 1285-1292. doi: $10.1093 / \mathrm{pcp} / \mathrm{pcf} 154$

Kang, Z., Huang, L., and Buchenauer, H. (2002). Ultrastructural changes and localization of lignin and callose in compatible and incompatible interactions between wheat and Puccinia striiformis. J. Plant Dis. Prot. 109, 25-37.

Kiss, E., Huguet, T., Poinsot, V., and Batut, J. (2004). The typA gene is required for stress adaptation as well as for symbiosis of Sinorhizobium meliloti 1021 with certain Medicago truncatula lines. Mol. Plant Microbe Interact. 17, 235-244. doi: 10.1094/MPMI.2004.17.3.235

Lalanne, E., Michaelidis, C., Moore, J. M., Gagliano, W., Johnson, A., Patel, R., et al. (2004). Analysis of transposon insertion mutants highlights the diversity of mechanisms underlying male progamic development in Arabidopsis. Genetics 167, 1975-1986. doi: 10.1534/genetics.104.030270

Li, C., Lin, H., and Dubcovsky, J. (2015). Factorial combinations of protein interactions generate a multiplicity of florigen activation complexes in wheat and barley. Plant J. 84, 70-82. doi: 10.1111/tpj.12960

Livak, K. J., and Schmittgen, T. D. (2001). Analysis of relative gene expression data using real-time quantitative PCR and the $2^{-\Delta \Delta C T}$ method. Methods 25, 402-408. doi: 10.1006/meth.2001.1262

Ma, J., Huang, X., Wang, X., Chen, X., Qu, Z., Huang, L., et al. (2009). Identification of expressed genes during compatible interaction between stripe rust (Puccinia striiformis) and wheat using a cDNA library. BMC Genomics 10:1. doi: 10.1186/1471-2164-10-586

Margus, T., Remm, M., and Tenson, T. (2007). Phylogenetic distribution of translational GTPases in bacteria. BMC Genomics 8:15. doi: 10.1186/1471-2164$8-15$

Mittler, R., Herr, E. H., Orvar, B. L., Van Camp, W., Willekens, H., Inzé, D., et al. (1999). Transgenic tobacco plants with reduced capability to detoxify reactive oxygen intermediates arehyper-responsive to pathogen infection. Proc. Natl. Acad. Sci. U.S.A. 96, 14165-14170. doi: 10.1073/pnas.96.24.14165

Mittler, R., Vanderauwera, S., Gollery, M., and Van Breusegem, F. (2004). Reactive oxygen gene network of plants. Trends Plant Sci. 9, 490-498. doi: 10.1016/j.tplants.2004.08.009

Mittler, R., Vanderauwera, S., Suzuki, N., Miller, G., Tognetti, V. B., Vandepoele, K., et al. (2011). ROS signaling: the new wave? Trends Plant Sci. 16, 300-309. doi: 10.1016/j.tplants.2011.03.007

Munns, R., and Tester, M. (2008). Mechanisms of salinity tolerance. Annu. Rev. Plant Biol. 59, 651-681. doi: 10.1146/annurev.arplant.59.032607.092911
Nilsson, J., and Nissen, P. (2005). Elongation factors on the ribosome. Curr. Opin. Struct. Biol. 15, 349-354. doi: 10.1016/j.sbi.2005.05.004

Owens, G. K., Kumar, M. S., and Wamhoff, B. R. (2004). Molecular regulation of vascular smooth muscle cell differentiation in development and disease. Physiol. Rev. 84, 767-801. doi: 10.1152/physrev.00041.2003

Petty, I. T., French, R., Jones, R., and Jackson, A. (1990). Identification of barley stripe mosaic virus genes involved in viral RNA replication and systemic movement. EMBO J. 9, 3453-3457.

Pfennig, P., and Flower, A. (2001). BipA is required for growth of Escherichia coli K12 at low temperature. Mol. Genet. Genomics 266, 313-317. doi: $10.1007 / \mathrm{s} 004380100559$

Polidoros, A., Mylona, P., and Scandalios, J. (2001). Transgenic tobacco plants expressing the maize Cat2 gene have altered catalase levels that affect plantpathogen interactions and resistance to oxidative stress. Transgenic Res. 10, 555-569. doi: 10.1023/A:1013027920444

Pugin, A., Frachisse, J. M., Tavernier, E., Bligny, R., Gout, E., Douce, R., et al. (1997). Early events induced by the elicitor cryptogein in tobacco cells: involvement of a plasma membrane NADPH oxidase and activation of glycolysis and the pentose phosphate pathway. Plant Cell 9, 2077-2091. doi: 10.2307/38 70566

Pumphrey, M., Bai, J., Laudencia-Chingcuanco, D., Anderson, O., and Gill, B. S. (2009). Nonadditive expression of homoeologous genes is established upon polyploidization in hexaploid wheat. Genetics 181, 1147-1157. doi: 10.1534/genetics.108.096941

Qi, S. Y., Li, Y., Szyroki, A., Giles, I. G., Moir, A., and O'connor, C. (1995). Salmonella typhimurium responses to a bactericidal protein from human neutrophils. Mol. Microbiol. 17, 523-531. doi: 10.1111/j.1365-2958.1995

Ramakrishnan, V. (2002). Ribosome structure and the mechanism of translation. Cell 108, 557-572. doi: 10.1016/S0092-8674(02)00619-0

Rea, D. K., Snoeckx, H., and Joseph, L. H. (1998). Late Cenozoic eolian deposition in the North Pacific: asian drying, Tibetan uplift, and cooling of the northern hemisphere. Paleoceanography 13, 215-224. doi: 10.1029/98PA00123

Robinson, V. L. (2008). Salmonella enterica serovar Typhimurium BipA exhibits two distinct ribosome binding modes. J. Bacteriol. 190, 5944-5952. doi: 10.1128/JB.00763-08

Scarpeci, T. E., Zanor, M. I., Carrillo, N., Mueller-Roeber, B., and Valle, E. M. (2008). Generation of superoxide anion in chloroplasts of Arabidopsis thaliana during active photosynthesis: a focus on rapidly induced genes. Plant Mol. Biol. 66, 361-378. doi: 10.1007/s11103-007-9274-4

Scheler, C., Durner, J., and Astier, J. (2013). Nitric oxide and reactive oxygen species in plant biotic interactions. Curr. Opin. Plant Biol. 16, 534-539. doi: 10.1016/j.pbi.2013.06.020

Scofield, S. R., Huang, L., Brandt, A. S., and Gill, B. S. (2005). Development of a virus-induced gene-silencing system for hexaploid wheat and its use in functional analysis of the Lr21-mediated leaf rust resistance pathway. Plant Physiol. 138, 2165-2173. doi: 10.1104/pp.105.061861

Sergiev, P. V., Lesnyak, D. V., Kiparisov, S. V., Burakovsky, D. E., Leonov, A. A., Bogdanov, A. A., et al. (2005). Function of the ribosomal E-site: a mutagenesis study. Nucleic Acids Res. 33, 6048-6056. doi: 10.1093/nar/gki910

Sharma, P., and Dubey, R. S. (2005). Drought induces oxidative stress and enhances the activities of antioxidant enzymes in growing rice seedlings. Plant Growth Regul. 46, 209-221. doi: 10.1007/s10725-005-0002-2

Shi, L. X., and Theg, S. M. (2013). The chloroplast protein import system: from algae to trees. Biochim. Biophys. Acta 1833, 314-331. doi: 10.1016/j.bbamcr.2012.10.002

Srivastava, S., and Dubey, R. (2011). Manganese-excess induces oxidative stress, lowers the pool of antioxidants and elevates activities of key antioxidative enzymes in rice seedlings. Plant Growth Regul. 64, 1-16. doi: 10.1007/s10725010-9526-1

Tamura, K., Peterson, D., Peterson, N., Stecher, G., Nei, M., and Kumar, S. (2011). MEGA5: molecular evolutionary genetics analysis using maximum likelihood, evolutionary distance, and maximum parsimony methods. Mol. Biol. Evol. 28, 2731-2739. doi: 10.1093/molbev/msr121

Torres, M. A. (2010). ROS in biotic interactions. Physiol. Plant. 138, 414-429. doi: 10.1111/j.1399-3054.2009.01326.x

Verstraeten, N., Fauvart, M., Versées, W., and Michiels, J. (2011). The universally conserved prokaryotic GTPases. Microbiol. Mol. Biol. Rev. 75, 507-542. doi: 10.1128/MMBR.00009-11 
Wang, C. F., Huang, L. L., Buchenauer, H., Han, Q. M., Zhang, H. C., and Kang, Z. S. (2007). Histochemical studies on the accumulation of reactive oxygen species (O2- and $\mathrm{H}_{2} \mathrm{O} 2$ ) in the incompatible and compatible interaction of wheat-Puccinia striiformis f. sp. tritici. Physiol. Mol. Plant Pathol. 71, 230-239. doi: 10.1016/j.pmpp.2008.02.006

Wang, F., Zhong, N. Q., Gao, P., Wang, G. L., Wang, H. Y., and Xia, G. X. (2008). SsTypA1, a chloroplast-specific TypA/BipA-type GTPase from the halophytic plant Suaeda salsa, plays a role in oxidative stress tolerance. Plant Cell Environ. 31, 982-994. doi: 10.1111/j.1365-3040.2008. 01810.x

Wang, X., Tang, C., Zhang, G., Li, Y., Wang, C., Liu, B., et al. (2009). cDNA-AFLP analysis reveals differential gene expression in compatible interaction of wheat challenged with Puccinia striiformis f. sp. tritici. BMC Genomics 10:289. doi: 10.1186/1471-2164-10-289

Wendel, J. F. (2000). Genome evolution in polyploids. Plant Mol. Biol. 42, 225-249. doi: 10.1007/978-94-011-4221-2_12

Wojtaszek, P. (1997). Mechanisms for the generation of reactive oxygen species in plant defence response. Acta Physiol. Plant. 19, 581-589. doi: 10.1007/s11738997-0057-y
Yu, X., Wang, X., Wang, C., Chen, X., Qu, Z., Yu, X., et al. (2010). Wheat defense genes in fungal (Puccinia striiformis) infection. Funct. Integr. Genomics 10, 227-239. doi: 10.1007/s10142-010-0161-8

Zurbriggen, M. D., Carrillo, N., and Hajirezaei, M. R. (2010). ROS signaling in the hypersensitive response: when, where and what for? Plant Signal. Behav. 5, 393-396. doi: 10.4161/psb.5.4.10793

Conflict of Interest Statement: The authors declare that the research was conducted in the absence of any commercial or financial relationships that could be construed as a potential conflict of interest.

Copyright (c) 2016 Liu, Myo, Ma, Lan, Qi, Guo, Song, Guo and Kang. This is an open-access article distributed under the terms of the Creative Commons Attribution License (CC BY). The use, distribution or reproduction in other forums is permitted, provided the original author(s) or licensor are credited and that the original publication in this journal is cited, in accordance with accepted academic practice. No use, distribution or reproduction is permitted which does not comply with these terms. 